\title{
Rethinking Strategies to Address Inequity in Immunization Services towards Achieving Universal Immunization Coverage (UIC) in Karachi, Pakistan
}

\author{
Iqbal Hossain ${ }^{1}$, Tariq Masood ${ }^{2}$, Akram Sultan ${ }^{3}$, Rana Safdar, Hamidreza Setayesh ${ }^{4}$, \\ Lora Shimp1, Soofia Younus4 \\ ${ }^{1}$ Immunization Center, JSI, Arlington, VA, USA \\ ${ }^{2}$ JSI, Karachi, Pakistan \\ ${ }^{3}$ EPI, Karachi, Pakistan \\ ${ }^{4}$ EPI, Islamabad, Pakistan \\ ${ }^{5}$ Gavi, Geneva, Switzerland \\ Email: ^Iqbal_hossain@jsi.com, soofiayunus@yahoo.com
}

How to cite this paper: Hossain, I., Masood, T., Sultan, A., Safdar, R., Setayesh, H., Shimp, L. and Younus, S. (2021) Rethinking Strategies to Address Inequity in Immunization Services towarsds Achieving Universal Immunization Coverage (UIC) in Karachi, Pakistan. World Journal of Vaccines, 11, 39-49.

https://doi.org/10.4236/wjv.2021.114006

Received: August 30, 2021

Accepted: October 5, 2021

Published: October 8, 202

Copyright $\odot 2021$ by author(s) and Scientific Research Publishing Inc. This work is licensed under the Creative Commons Attribution International License (CC BY 4.0).

http://creativecommons.org/licenses/by/4.0/ (c) (i) Open Access

\begin{abstract}
Karachi, the largest city in Pakistan, having high population growth and a complex health care environment, has highest density of unimmunized (zero dose) and under-immunized children. The main reasons of low immunization coverage in Karachi were lack of governance and accountability in a duplicative and fragmented health management structure, weak and inequitable immunization services, and lack of demand and trust among people for immunization services. The Expanded Programme on Immunization (EPI), Ministry of Health $(\mathrm{MOH})$ in Sindh Province spearheaded a structured and collaborative process to develop strategies for addressing inequity in immunization services towards achieving Universal Immunization Coverage (UIC) in Karachi. The process included a situation analysis with gathering quantitative and qualitative information on the root causes of zero-dose and inequity of the immunization services. The strategies and interventions were developed with multi-layer input and feedback of the stakeholders and partners, and focusing primarily to address gaps in three program areas: governance, leadership and accountability; immunization service delivery; and building demand and trust among the people. The interventions were further prioritized for high-risk areas; identified based on maximum number zero-dose children, presence of large slum areas, measles outbreak and on-going circulation of wild poliovirus. Finally, costing for the Roadmap activities was done through consultation with partners and aligning domestic and external (donor) re-
\end{abstract}


sources. In this paper, we have highlighted the unique process the Sindh Government undertook in collaboration with the stakeholders and partners to develop strategies and interventions for addressing inequity in urban immunization services in Karachi towards achieving Universal Immunization Coverage (UIC). Similar processes can be adapted, as a potential model, for developing strategies to achieve universal health coverage in the cities of Pakistan and in other countries.

\section{Keywords}

Urban Immunization, Inequity, Strategies, Roadmap, Universal Immunization Coverage

\section{Background}

Pakistan is urbanizing rapidly, with about $36.4 \%$ of the national population currently residing in urban areas [1]. UN Population Division estimates that nearly half of Pakistan's population will live in cities by 2025, and Sindh is among the most urbanized province in Pakistan with over half of its population (52\%) living in urban areas [2]. Karachi, the capital of Sindh province, has a population of over 16 million, making it the largest city in Pakistan [3]. Nearly half (7 million, $45.5 \%)$ of Karachi's population reside in 988 slum areas [4]. People of various linguistic and religious origins from different parts of the country migrate to Karachi for economic, educational, and political opportunities.

The health management structure in Karachi is complex and duplicative with infrastructure and providers from multiple sources (Figure 1). Karachi has got highest density of unimmunized (zero dose) and under-immunized children (more than 400,000 in 2018) in Pakistan [5]. The public health system in Karachi could not keep pace with the rapidly growing urban population, and people living in unauthorized urban settlement areas are excluded from immunization services resulting in inequity of immunization coverage. The Pakistan Demographic and Health Survey (PDHS 2017-18) found full immunization coverage with basic vaccines ${ }^{1}$ in the urban areas of the Sindh province at $66 \%$, with major inequity in coverage between the richest (80\%) and poorest $(38 \%)$ people in both rural and urban populations [6]. The Multiple Indicator Cluster Survey, 2014 [7] found low immunization coverage in all districts of Karachi, with lowest coverage in the peri-urban West (45\%) and Malir (48\%) districts (Figure 2). Due to the presence of a large number of unimmunized and under-immunized children, Karachi experiences frequent outbreaks of measles, and wild poliovirus continues to circulate in the city [8] [9]. In addition, Karachi and urban Sindh have the highest infant and under-5 mortality rates, 50 and 56 per 1000 live births, respectively, when compared to other provinces in Pakistan [6].

${ }^{1}$ One dose of BCG, 3 doses of Pentavalent vaccine, 3 doses of oral polio vaccine, one dose of measles vaccine. 


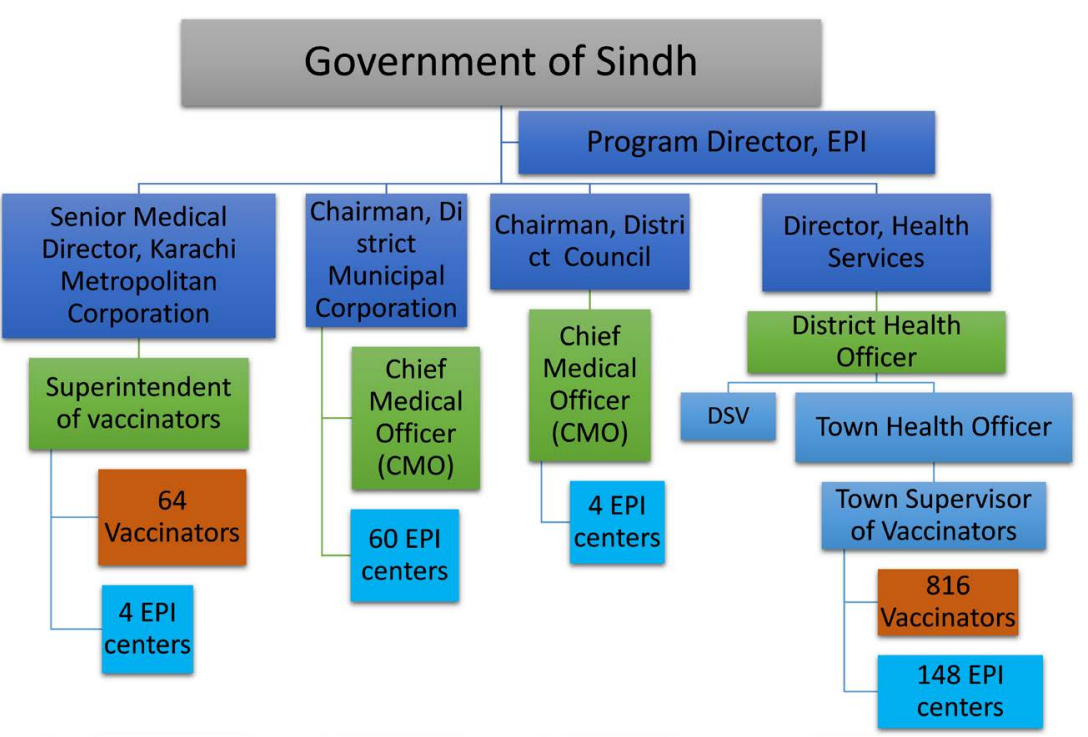

Figure 1. Health program management structure in Karachi city.

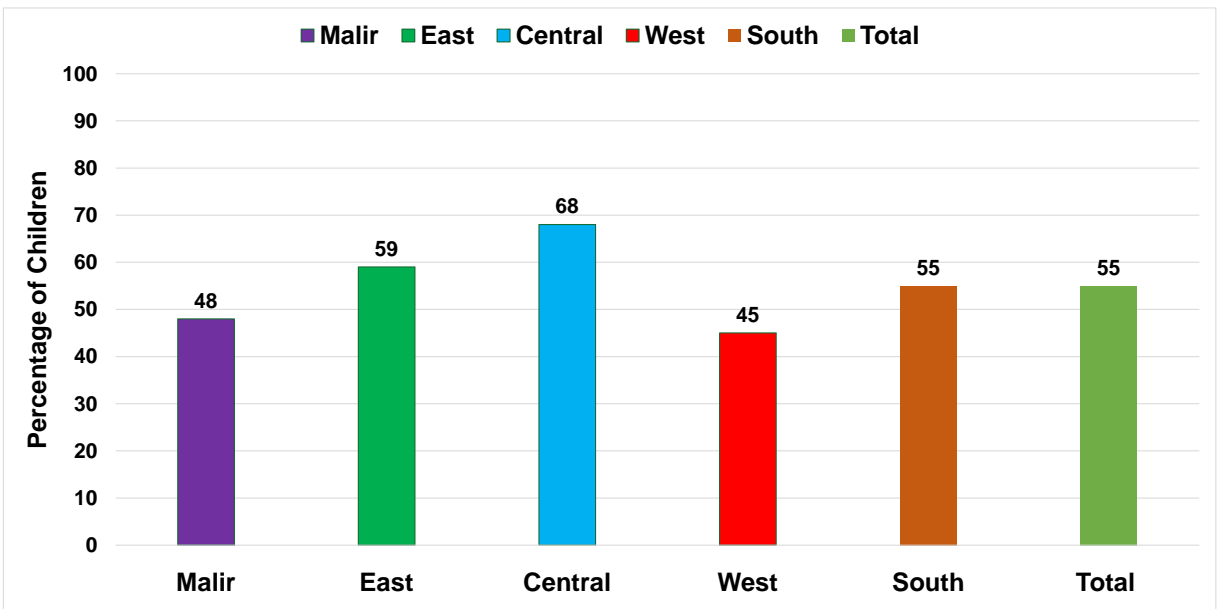

Figure 2. Full immunization coverage in Karachi, by district (Source: Multi-indicator Cluster Survey (MICS) 2014).

Achieving Universal Immunization Coverage (UIC) has been a commitment of both the Federal and Sindh Provincial Governments. In order to achieve UIC the Sindh EPI initiated a collaborative process involving stakeholders and partners to develop strategies for a Roadmap for improving equitable access of immunization services to all population of Karachi regardless of where they live.

\section{The Steps and Processes of Strategy Development for the Roadmap}

The Roadmap strategy development was a stepwise co-creation process with multiple layers of stakeholders' input and feedback for developing evidence-based interventions for addressing gaps and weaknesses of the routine immunization services as described below (Figure 3).

\section{Step 1: Situational analysis}




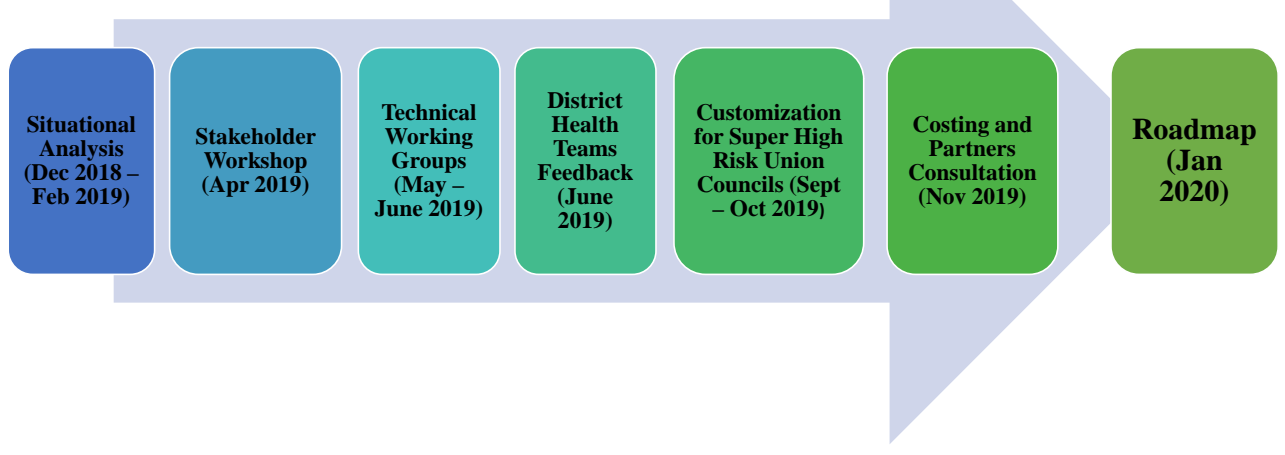

Figure 3. Roadmap development process.

The Roadmap development process began with a situational analysis (December 2018-February 2019) comprised of a review of existing literatures, and a mixed methods data collection (quantitative and qualitative) to identify gaps, barriers and challenges of routine immunization services in the context of zero-dose and inequity of services. Quantitative data collection included randomly selected household surveys in the slum areas of the six select union councils, the lowest administrative unit, to find out access and utilization of immunization services. In addition, remote Geographic Information System (GIS) mapping was done to visualize the distribution of EPI centers, vaccinators, and cold chain points at the Union Council and surrounding peri-urban areas of the city. Qualitative data collection in the form of 115 key informant interviews was done with a range of stakeholders from different levels of the health system (vaccinators, lady health workers, community health workers, private practitioners, academic institutions, and members of civil society organization) to understand provider's barriers and challenges. In addition, 27 focus group discussions were conducted with caregivers of zero-dose and partially immunized children to understand their barriers to access the immunization services. Data collection protocol and tools were reviewed and approved by the Institutional Review Board (IRB) before commencing data collection, and strict confidentially of participants was maintained during data collection, analysis, and report writing.

The situation analysis revealed that due to duplicative health management infrastructure in Karachi there are governance, accountability, and program coordination issues with lack of supervision and monitoring of the immunization program. GIS mapping indicated weak immunization service delivery with gross shortage of EPI centers and vaccinators in some areas (Figure 4). FGD with caregivers revealed widespread fears and mistrust among people about immunization with a range of reasons including low-quality of services, lack of information, fear of side effects, rumors and lack of motivation (Figure 5).

Step 2: Workshop for the stakeholders to develop strategy

A two-day workshop was organized with participation of a diverse group of 
stakeholders including federal and provincial EPI teams, provincial Emergency Operations Cell (EOC), district and town officers, and representatives from local government bodies, private sector entities, non-governmental organizations (NGOs), and civil society organizations (CSO) in April 2019. The findings of the situation analysis were shared in the workshop to co-create strategies for strengthening governance, leadership and accountability; strengthen delivery of immunization services in all areas, and to increase trust and demand of immunization services among people.

Steps 3: Technical Working Groups (TWGs) for development of interventions

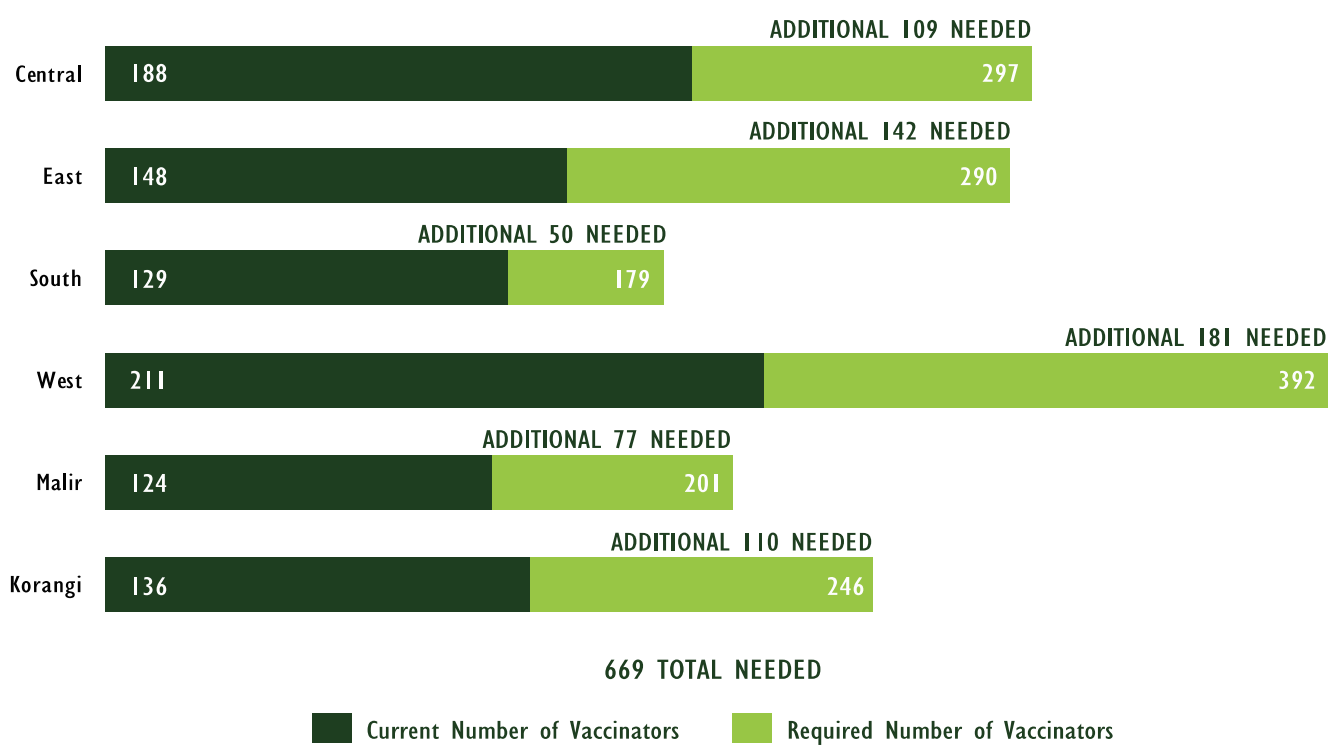

Figure 4. Number of existing vaccinators compared to required number of vaccinators.

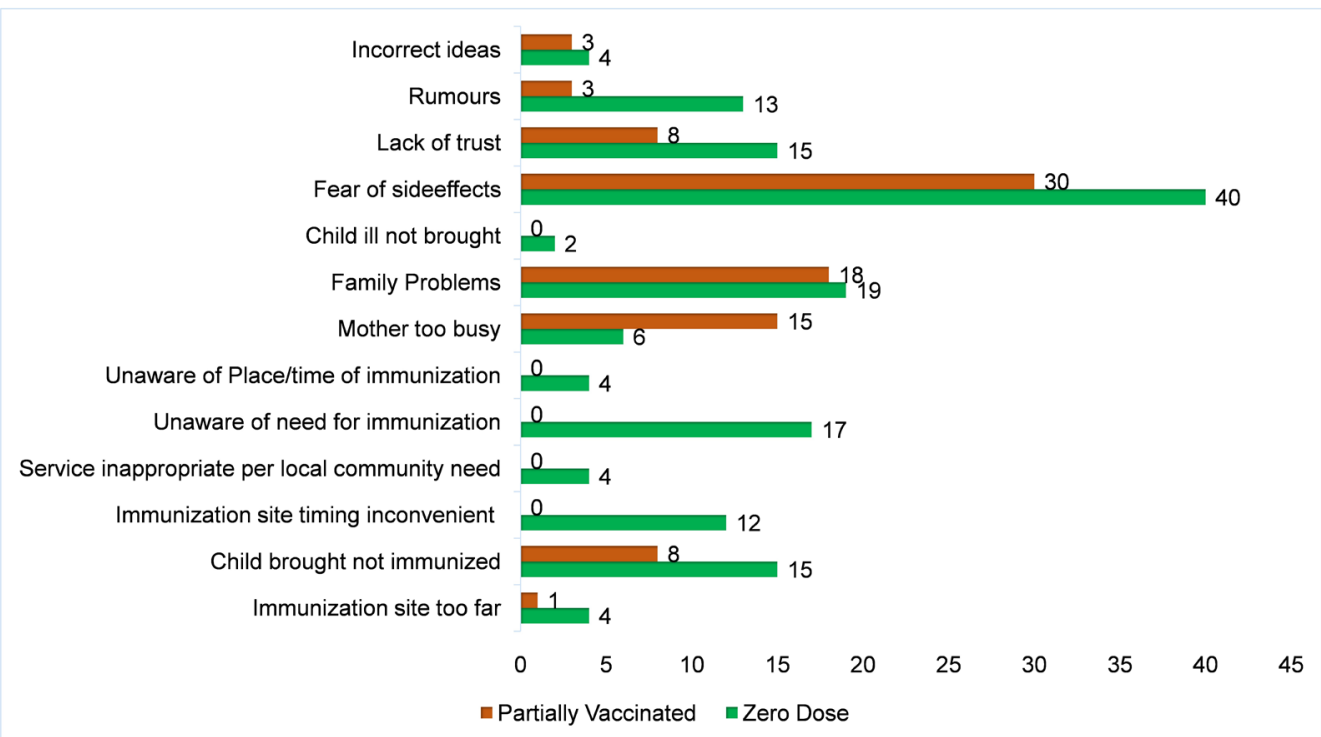

Figure 5. Reasons given by caregivers of zero dose and partially vaccinated children (Multiple reason accepted). 
Three TWG were formed in the stakeholders' workshop with representatives from Sindh EPI and EOC, Federal EPI, and key partners to develop interventions in each of the three strategic areas in May-June 2019. Each TWG had a series of consultative meetings to develop, review, refine and finalize the interventions for each of the three strategic area (as described in the box below).

Box 1: Interventions to improve governance, leadership and accountability

- Establish and strengthen governance platforms from provincial to lowest administrative (union) levels

- Develop unified and robust management structure at all levels

- Build capacity of management staff to optimally perform management functions

- Strengthen functional linkages and coordination between the stakeholders (Sindh EPI, Karachi municipal authority, NGOs, Private sector, Civil Society etc)

- Conduct oversight and program monitoring of immunization services for accountability

- Improve synergies between polio eradication initiatives (PEI) and routine immunization (RI)

\section{Box 2: Interventions to improve delivery of immunization services}

- Expand EPI services with increase in number and quality of services by refurbishing the sites and rational placement of vaccinators

- Expand reach and quality of outreach services in the slum through joint microplanning and integration of filed workers of all departments

- Arrange evening/weekend RI services (fixed site or outreach) for children of working class parents in the slum areas.

- Develop a private sector engagement strategy to provide immunization services in areas where there is no public health facility/health workers available e.g. memorandum of understanding (MOU) for public-private partnership

- Minimize missed opportunity of vaccination by providing daily vaccination in facilities with high load of clients and establishing referral system between child health department and vaccination sites.

\section{Box 3: Interventions to improve trust and demand among caregivers}

- Hire communication officers at provincial and district level to develop and disseminate communication messages and materials

- Conduct special communication activities in high risk areas with messages in appropriate languages and means of communication

- Involve local community leaders, indigenous leaders, and religious leaders for social mobilization and message dissemination on immunization

- Train health workers on interpersonal communication

\section{Step 4: Feedback from the District Health Team (DHT)}

The proposed strategies and the interventions were shared in June 2019 with the District Health Team (DHT) in two districts (Central and East) for their feedback, and their inputs were incorporated into the Roadmap interventions.

Step 5: Customization of interventions for the Super High Risk Union 


\section{Council (SHRUCs)}

The interventions were customized further in subsequent workshops in September-October 2019 for eight prioritized SHRUCs identified based on maximum number zero-dose children, presence of large slums, measles outbreaks and on-going circulation of wild poliovirus. Tailored action plans were developed for reaching zero-dose and under-immunized children, reducing dropout rates and missed opportunities of vaccination.

\section{Step 6: Costing for the Roadmap activities}

Costing for the Roadmap was done through a series of meetings in November 2019 with representatives from the Federal and Provincial EPI and key partners for implementation of the Roadmap interventions, and for aligning available domestic and donor for three years (2020-2022).

\section{Discussion}

\subsection{Multi-Step and Co-Creation Process for Developing Strategies and Interventions for the Roadmap}

The embodied multi-steps and co-creation process for development of Karachi Roadmap brought in the expertise and experience of many stakeholders from various perspectives. Each step informed the activities being done in the next step of the process towards the development of a comprehensive product (the Roadmap). The iterative process with different layers of feedback and inputs from the diverse stakeholders was unique. The co-creation process brought overwhelming support and buy-in of the stakeholders and partners for development of the Roadmap and its implementation. In other settings in Asia, the deliberate inclusion and engagement of stakeholders while planning public health program strategy positively impacted the program design [10] [11] [12].

\subsection{Addressing Growing Inequity to Improve Urban Immunization Services}

More than $90 \%$ urban growth over the next 40 years will occur in developing countries where an estimated one third (nearly one billion people) of the urban population lives in poverty [13], which will lead to inequity with increased number of zero-dose and under-vaccinated population in the urban areas leading higher potential for transmission of vaccine preventable diseases (VPDs) in the densely populated urban areas [14], and resulting high morbidity and mortality [15]. Regardless of the comparative advantage of having multiple service provisions available from different sources, urban poor people have limited access to health care and immunization services due to economic and social reasons including inability to pay, unsuitable location of the facilities, social distance, inconvenient hours of operation, and poor quality of immunization services, and health workers may be reluctant to work in the unsafe and dirty slum environment. Urban poor people are further disadvantaged as both parents have to work outside for income to bring food for the family - a limitation for vaccinating their kids during regular 
immunization session hours. Immunization inequality in urban areas are created due to either exclusion (in which most of the populations do not have access to services), or due to marginalization in which only the poor are excluded [16]. The Expanded Program on Immunization (EPI) was designed over forty years ago as a rural model and has not been adjusted to meet the needs of the growing urban population. The children of rural-urban migrant families are less likely to be fully immunized than urban non-migrants and the general population in low-income and middle-income countries (LMICs) [17]. On the other hand, the issue of inequity in urban areas may remain hidden with overall high coverage in the city. Innovative approach is necessary to uncover the inequity in urban immunization services. In Karachi, in addition to household survey, visualization through GIS technology of existing immunization services outlets and placement of vaccinators was useful tool to reveal inequity of immunization services, which may have been more difficult to identify if the data were presented in a tabular way. There was also under-utilization of existing private sector facilities for immunization services in the areas where there is no infrastructure from public health system. These information were helpful to justify the strategies to improve delivery of immunization services: short-term strategy with extended hours of services for working urban poor parents/caregivers and involving private sector health facilities to provide immunization services; medium term strategy such as hiring more vaccinators and their equitable distribution; and long-term strategy such as building more public health facilities for urban poor settlements and peri-urban areas. Sindh Provincial Government that has already taken some of these initiative for addressing inequity in immunization services and leveraging resources for implementation of the interventions in Karachi. In other immunization research, engagement of a diverse set of stakeholders including public-private partnerships demonstrated opportunities that included sharing of resources, technical assistance and best practices [18].

\subsection{Building Trust and Demand for Immunization Services}

Urban populations are more complex and socially heterogeneous than rural populations, comprising different ethnicities, social structures, cultural beliefs, and lack in community cohesion or resilience [19]. Health message do not spread in urban areas as easily as in the rural areas. FGD with caregivers of zero-dose and under-vaccinated children revealed multiple communication challenges leading to under-utilization of immunization services such as fear of side effects, being too busy, and not knowing that children must receive multiple doses for full protection, and working parents reported about inconvenient clinic hours. Still, communities were generally found receptive to vaccination services. Roadmap included interventions to build community trust and demand for the services including involvement of local traditional and religious leaders. In addition, alternative immunization service delivery provisions (evening and/or weekend immunization session) was included for children of working parents who are not able to utilize services during the regular sessions hours. In Bangladesh, such com- 
munication initiatives and extended hours of immunization services (evening services) was found to have significantly improve the full immunization coverage among children of working parents in the slum areas [20].

\subsection{Recognizing the Resource Needs for Implementation of the Roadmap Interventions}

The Karachi Roadmap development showed great promise. However, the successful implementation of the Roadmap interventions requires resource mobilization at all levels including domestic funding for immunization, as well as external resources to cover gaps, while simultaneously leveraging and exploring financing resources for alternative provision of services through extended hours or at the weekend. The Karachi Roadmap costing initiative can act as an example to follow in this regard. In Indonesia, effective multi-stakeholder coordination at the provincial level worked to garner increased mobilization of technical and financial resources from both domestic and international partners [12].

\section{Conclusion}

The Karachi Roadmap development initiative is an example of a collaborative and participatory multi-step process between a variety of stakeholders to align support, decrease inequities and overcome implementation barriers of immunization services. Development of Roadmap prompted the Sindh government to take initiatives to strengthen immunization system in Karachi such as hiring more vaccinators, refurbishing public health facilities as vaccination centers, providing immunization services in the evening for the children of working parents, and developing memorandum of understanding (MOU) to involve private sector health facilities to provide immunization services in areas where there is no immunization services from the public health system. The scale-up of the similar initiative to different cities throughout Pakistan, and replicating the process for achieving universal health coverage (UHC) will rely on the flexibility of the strategy and the commitment from stakeholders' for engagement in the planning, implementation and monitoring processes.

\section{Acknowledgements}

The authors would like to thank the health workers in Karachi for their in-sights, the caregivers for providing valuable information during the situation analysis, and the stakeholders and partners for their contribution during the Roadmap development process. Finally, sincere appreciation to Gavi, the Vaccine Alliance, for the financial support without which the development of Karachi Roadmap would not be possible.

\section{Conflicts of Interest}

The authors declare no conflicts of interest regarding the publication of this paper. 


\section{References}

[1] Pakistan Bureau of Statistics, Government of Pakistan (2017) 2017 Census of Pakis$\tan$. https://www.pbs.gov.pk/content/final-results-census-2017

[2] Development Advocate Pakistan: Sustainable Urbanization (2018). https://www.pk.undp.org/content/pakistan/en/home/library/development_policy/d ap-vol5-iss4-sustainable-urbanization.html

[3] Karachi Population (2020) World Population Review. https://worldpopulationreview.com/world-cities/Karachi-population

[4] Ministry of Climate Change, Government of Pakistan (2015) National Report of Pakistan for HABITAT III, Islamabad.

[5] WHO UNICEF Immunization Coverage Estimates 2018 Revision (2019). https://www.who.int/immunization/monitoring surveillance/routine/coverage/WU ENIC notes.pdf?ua $=1$

[6] National Institute of Population Studies (NIPS) (2019) Pakistan Demographic and Health Survey (PDHS 2017-18). NIPS and ICF, Islamabad, Pakistan, and Rockville, Maryland, USA.

[7] Sindh Bureau of Statistics and UNICEF (2015) Sindh Multiple Indicator Cluster Survey 2014, Final Report, Karachi.

[8] Pakistan Polio Snapshot-July 2020 (2020). http://polioeradication.org/news-post/pakistan-polio-snapshot-july-2020/

[9] WHO|Measles-Global Situation (2020). https://www.who.int/health-topics/measles\#tab=tab 1

[10] Archana, S., Karmacharya, B.M., Rashmi, M., et al. (2019) Stakeholder Engagement in Planning the Design of a National Needs Assessment for Cardiovascular Disease Prevention and Management in Nepal. Global Heart, 14, 181-189. https://doi.org/10.1016/j.gheart.2019.05.002

[11] Soeung, S., Grundy, J., Kamara, L., McArthur, A. and Samnang, C. (2007) Developments in Immunization Planning in Cambodia-Rethinking the Culture and Organization of National Program Planning. Rural Remote Health, 7, Article No. 630. https://doi.org/10.22605/RRH630

[12] Kurniati, A., Rosskam, E., Afzal, M.M., Suryowinoto, T.B. and Mukti, A.G. (2015) Strengthening Indonesia's Health Workforce through Partnerships. Public Health, 129, 1138-1149. https://doi.org/10.1016/j.puhe.2015.04.012

[13] World Bank (2008) Urban Poverty: A Global View. https://documents1.worldbank.org/curated/en/954511468315832363/pdf/430280N WP0Glob10Box327344B01PUBLIC1.pdf

[14] Tucker, J.D., Hao, C., Zou, X., et al. (2013) The Influence of Migration on the Burden of and Response to Infectious Disease Threat in China: A Theoretically Informed Review. United Nations Research Institute for Social Development (UNRISD).

[15] Taylor, W.R., Ruti-Kalisa, Ma-Disu, M. and Weinman, J.M. (1988) Measles Control in Urban Africa Complicated by High Incidence of Measles in the First Year of Life. American Journal of Epidemiology, 127, 788-794. https://doi.org/10.1093/oxfordjournals.aje.a114860

[16] Matthews, Z., Channon, A., Neal, S., Osrin, D., Madise, N. and Stones, W. (2010) Examining the "Urban Advantage" in Maternal Health Care in Developing Countries. PLoS Medicine, 7, e1000327. https://doi.org/10.1371/journal.pmed.1000327 
[17] Awoh, A.B. and Plugge, E. (2015) Immunization Coverage in Rural-Urban Migrant Children in Low and Middle-Income Countries (LMICs): A Systemic Review and Meta-Analysis. Journal of Epidemiology and Community Health, 70, 305-311. https://doi.org/10.1136/jech-2015-205652

[18] Bennett, S., Mahmood, S.S., Edward, A., Tetui, M. and Ekirapa-Kiracho, E. (2017) Strengthening Scaling up through Learning from Implementation: Comparing Experiences from Afghanistan, Bangladesh and Uganda. Health Research Policy and Systems, 15, Article No. 108. https://doi.org/10.1186/s12961-017-0270-0

[19] Harpman, T. (1986) Health and the Urban Poor. Health Policy and Planning, 1, 5-18. https://doi.org/10.1093/heapol/1.1.5

[20] Uddin, M.J., Larson, C.P. and Oliveras, E. (2010) Effectiveness of Combined Strategies to Improve Low Coverage of Child Immunization in Urban Slums of Bangladesh. Health Policy and Planning, 25, 50-60. https://doi.org/10.1093/heapol/czp041 